\title{
GUI BASED PREDICTION OF HEART STROKE STAGES BY FINDING THE ACCURACY USING MACHINE LEARNING ALGORITHM
}

\author{
Dr. J.C. Kavitha \\ Assistant Professor, Department of Information Technology, \\ RMD Engineering College, Tamil Nadu, India \\ K. Jayasurya, Kurakula Premkrishna, R. Praveenkumar \\ Student, Department of Information Technology, \\ RMD Engineering College, Tamil Nadu, India
}

\begin{abstract}
Numerous prescient strategies have been broadly applied in clinical basic leadership, for example, anticipating event of a malady or analysis, assessing forecast or result of illnesses and helping clinicians to prescribe treatment of ailments. Be that as it may, the traditional prescient models or methods are as yet not powerful enough in catching the hidden information since it is unequipped for reproducing the multifaceted nature on include portrayal of the therapeutic issue spaces. To beat this issue, prescient logical methods for heart stroke utilizing AI model applied on given clinic dataset. The atrial fibrillation manifestations in heart patients are a significant hazard factor of stroke and offer regular factors to anticipate stroke and the examination of given dataset by administered AI calculation to catch a few data resembles, variable distinguishing proof, uni-variate investigation, bi-variate and multi-variate examination, missing worth medicines and so on. The primary target is to prescient examination model to analyze heart stroke phases of patients. Furthermore, talk about the exhibition from the given clinic dataset with assessment of grouping report and recognize the disarray framework. To propose an AI based strategy to precisely foresee the heart stroke by given characteristics as best exactness from contrasting administer characterization AI calculations. Moreover, to think about and examine the exhibition of different AI calculations from the given social insurance division dataset with assessment grouping report, distinguish the disarray lattice and to sorting information from need and the outcome shows that the viability of GUI based the proposed AI calculation system can be contrasted and best exactness with accuracy, Recall and F1 Score.
\end{abstract}

Key words: Dataset, python, Prediction of Accuracy result 
Cite this Article: Dr. J.C. Kavitha, K. Jayasurya, Kurakula Premkrishna, R. Praveen kumar, GUI Based Prediction of Heart Stroke Stages by Finding the Accuracy Using Machine Learning Algorithm, International Journal of Computer Engineering and Technology 10(6), 2019, pp. 41-50.

http://iaeme.com/Home/issue/IJCET?Volume $=10 \&$ Issue $=6$

\section{INTRODUCTION}

Computer based intelligence is to anticipate the future from past data. Simulated intelligence (ML) is a kind of automated thinking (AI) that enables PCs to learn without being unequivocally altered. Man-made intelligence bases on the progression of Computer Programs that can change when displayed to new data and the essentials of Machine Learning, utilization of a fundamental AI estimation using python. Technique of getting ready and estimate incorporates use of explicit figuring. It feed the readiness data to a count, and the computation uses this planning data to give conjectures on another test data. Computer based intelligence can be commonly disconnected in to three characterizations. There are coordinated learning, independent learning and bolster learning. Overseen learning program is both given the data and the relating stamping to learn data must be named by an individual already. Solo learning is no imprints. It provided for the learning computation. This estimation needs to understand the gathering of the data. Finally, Reinforcement adjusting continuously helps out its condition and it gets positive or negative contribution to improve its introduction.

\section{RELATED WORK}

Controlled Machine Learning is the vast majority of sensible AI uses oversaw learning. Coordinated learning is the spot have input factors $(\mathrm{X})$ and a yield variable $(\mathrm{y})$ and use an estimation to take in the mapping limit from the commitment to the yield is $y=f(X)$. The goal is to construe the mapping limit so well that when you have new data $(\mathrm{X})$ that you can envision the yield factors (y) for that data. Frameworks of Supervised Machine Learning computations join key backslide, multi-class course of action, Decision Trees and support vector machines, etc. Managed learning requires that the data used to set up the figuring is currently set apart with right answers. Directed learning issues can be moreover assembled into Classification issues. This issue has as target the improvement of a short model that can anticipate the estimation of the dependent quality from the characteristic components. The qualification between the two assignments is how the poor characteristic is numerical for out and out for game plan. A portrayal model undertakings to arrive at some assurance from watched regards. Given at any rate one information sources a request model will endeavor to anticipate the estimation of at any rate one outcomes. A gathering issue is where the yield variable is a class, for instance, "red" or "blue".

\section{LITERATURE SURVEY}

TITLE: Breast tumor detection using empirical mode decomposition features

AUTHOR: Hongchao Song, Aidong Men and Zhuqing Jiang

YEAR: 2017

\section{DESCRIPTION:}

Head section examination (PCA) is one of the most extensively used segment extraction strategies; regardless, PCA is conversely influenced by signal misalignment. Observational mode breaking down (EMD)- based component extraction system that is progressively overwhelming to hail misalignment. The accurate features are expelled from the rotted sub 
gatherings of the primary sign. The exploratory results got from clinical data show that the acknowledgment exactness is improved by the mix of features from EMD and PCA. We were convinced to explore the usage of EMD features because of their potential life to the system jitter that is customary in microwave chest dangerous development looks at. We evaluated the recognizable proof execution using EMD-based features and the customarily used PCA features using clinical primer data accumulated over an eight-month time allotment got together with a numerical tumor response improvement system. We saw that the usage of features subject to EMD prompts on a very basic level improved revelation execution appeared differently in relation to PCA-based features. The inspiration for microwave-based bosom tumor identification is that huge contrasts in the dielectric properties exist among harmful and solid bosom tissues in the microwave recurrence run. The assessed harmful totypical bosom tissue differentiate is roughly 2:1 to 10:1 contingent upon the thickness of the ordinary tissue. AI calculations are utilized to catch the distinctions that exist among ordinary and threatening tissues. Highlight extraction is a significant advance in accomplishing great bosom disease recognition execution. Misalignment among bosom examines, which is brought about by hardware development and the framework's inborn jitter in the clock and testing oscilloscope, brings about various highlights being extricated from various sweeps of a similar patent. Along these lines, making highlights that are obtuse toward the time move existing among checks is significant.

TITLE: A multimodal deep neural network for human breast cancer prognosis prediction by integrating multi-dimensional data

AUTHOR: Dongdong Sun, Minghui Wang

YEAR: 2018

\section{DESCRIPTION:}

Chest threatening development is the most significantly powerful ailment and a huge medicinal issue in females, and a principle wellspring of illness related passings around the globe. to the limit of foreseeing threatening development surmise even more unequivocally not solely could help chest infection patients consider their future, yet likewise help clinicians with choosing instructed decisions and further guide appropriate treatment. In the meantime, gauge expect a critical activity in clinical works for all clinicians, particularly those clinicians working with flashing survivor. Right when a reasonably accurate estimation of expectation is available, clinicians routinely use surmise desire data to help with clinical essential authority, develop patients' capability for care designers, structure and examination of clinical fundamentals. To comprehensively survey our proposed methodology, we use multiple times cross endorsement investigate in consistent with past existing examinations of harmful development perception figure. Specifically, the patients in our preliminary are randomized into ten subsets. For each cycle, nine of those ten subsets are furthermore isolated into getting ready $(80 \%)$ and endorsement $(20 \%)$ sets, while the remaining one subset is utilized as testing set. Thusly, we gain the desire scores of each testing subset after ten changes and a short time later combine them as a general figure scores. Likewise, in our study, MDNNMD does not optimize the model configurations and weight coefficients simultaneously. For performance evaluation, we plot receiver operating characteristic (ROC) curve, which shows the interplay between sensitivity and 1-specificity by varying a decision threshold, and computes the AUC. The evaluation metric, Sensitivity (Sn), Specificity (Sp), Accuracy (Acc), Precision (Pre) and Matthew's correlation coefficient (Mcc) are also used for performance evaluation. We compare the performance of MDNNMD with three widely used methods for prognosis prediction of breast cancer: SVM, RF and LR. Ten-fold cross validation experiment for 
prognosis prediction of breast cancer is conducted with four different methods. In this study, we use an RF and LR package obtained from scikitlearn. Breast cancer is the most common disease and is usually associated with poor prognosis. In this work, we present a novel multimodal deep neural network by integrating multi-dimensional data named MDNNMD to predict the survival time of human breast cancer. To efficiently incorporate multidimensional data including gene expression profile, CNA and clinical data in breast cancer, three independent DNN models are constructed to generate a final multimodal DNN model considering the heterogeneity of different types of data.

TITLE: Clinical Pilot Application of Super-goals US Imaging in Breast Cancer AUTHOR: S. Dencks, M. Piepenbrock and T. Opacic

YEAR: 2018

\section{DESCRIPTION:}

To consider the outcomes of top tier separate moved up to super-objectives US imaging and proficiently analyze the estimations to get signs for the improvement of picture obtainment and getting ready in future clinical examinations. In such way, the use of a submersion model to the reproduced vessels is exhibited to be a significant gadget not only to measure the estimation times essential to acceptably imitate the microvasculature yet moreover for the endorsement of the estimations. The parameters from this model can in like manner serve to streamline separate administrator center and implantation shows. The clinical excitement for the microvasculature of tissues is unpredictable since deviations from average vessel improvement expect a vocation in different sicknesses, as flammable or ulcerative issue, dangerous development, or blinding eye diseases. Particularly for tumors, it is understood that their vascularization is morphologically bizarre also, that features like the tortuosity of vessels, their fanning, their sporadic vessel estimations, and the inhomogeneity all through the tumor contain fundamental information on its forcefulness. Valuable parameters like a chance to-top, top improvement and upslope of normal time-power (TI) twists are confined to an overall explanation of perfusion. Furthermore, their assessment is inconvenient and dishonest at the single voxel level. By revealing vascular features at super-objectives and assessing even outstandingly low stream rates of single vessels, ULM is depended upon to fundamentally improve the differential discovering, surmise, and the checking and desire for treatment responses. Nevertheless, the ability of ULM is unequivocally interrelated with the particular believability in a clinical set-up.

TITLE: Data Mining Techniques to Detect Motor Fluctuations in Parkinson's disease

AUTHOR: Paolo Bonato, Delsey M. Sherrill, David G. Standaert

YEAR: 2004

\section{DESCRIPTION:}

To identify motor patterns of primary and secondary movement disorders in PD, such as tremor, rigidity, dyskinesia, akinesia, and dystonia in a manner that is both objective and automatic. In addition to successfully classifying these disorders for the purpose of assessment, data mining has the potential of increasing our understanding of these disorders. Surprisingly little information is available today in the research literature on the study and comparison of specific motor patterns from the full complement of movement disorders in advanced $\mathrm{PD}$, and on factors that contribute to variability among patients and within the same patient over time. 
TITLE: Detecting Parkinsons' Symptoms in Uncontrolled Home Environments: A Multiple Instance Learning Approach

AUTHOR: Samarjit Das, Breogan Amoedo, Fernando De la Torre

YEAR: 2012

\section{DESCRIPTION:}

To demonstrated the use of multiple instance learning for detecting PD motor symptoms in uncontrolled home environments. Our work addressed the formulation of PD symptom detection from weakly labeled data as a semi-supervised multiple instance learning problem. The features were carefully chosen to address the subject and symptom specific nature of the problem. We show promising preliminary results on four days of monitoring performed with two PD subjects. In future work, we plan to increase our subject pool and utilize optimal feature selection strategies under MIL frameworks for developing robust person-specific models. These techniques can potentially be adapted to various other physiological sensing and monitoring applications as well.

\section{EXSISTING SYSTEM}

Hindrances in available expressive estimations limit the sufficiency of directing medications for cardiogenic stagger. In current clinical practice, cardiovascular state is assembled through estimation of aspiratory slim wedge weight and reliance on direct approximations among weight and stream to evaluate periphery vascular resistance. Mechanical circulatory assistance contraptions living inside the left ventricle and aorta allow to both choosing cardiovascular and vascular state and offering helpful bit of leeway. it impact the controllable technique for movement and transvalvular position of a possessing percutaneous ventricular assistance contraption to assess vascular and, in this manner, cardiovascular state through the effects of device vein coupling across over different degrees of device support. Systems: Vascular state is constrained by evaluating changes in the weight waveforms began through purposeful combination in the contraption created blood stream. It assesses this effect by applying a lumped parameter model to measure state-explicit vascular obstruction and consistence and compute beat-to-beat stroke volume and cardiovascular yield in both creature models and review understanding information without outer adjustment. Results: Vascular state was precisely anticipated in patients and animalsin both benchmark and test conditions, stroke volume was anticipated inside an all-out RMS blunder of $3.71 \mathrm{~mL}(\mathrm{n}=482)$. End: it shows that gadget blood vessel coupling is an incredible asset for assessing patient and state explicit parameters of cardiovascular capacity. Hugeness: it bits of knowledge may yield improved clinical consideration and bolster the advancement of cutting edge mechanical circulatory help gadgets that decide and work pair with the upheld organ.

\section{PROPOSED SYSTEM}

\section{Exploratory Data Analysis}

It will be using Jupyter notebook to work on this dataset and will first go with importing the necessary libraries and import our dataset to Jupyter notebook:

- Problem Define

- Preparing Data

- Evaluating Algorithms

- Improving Results

- Prediction the Result 


\section{Splitting the Dataset}

The information use is generally part into preparing information and test information. The preparation set contains a known yield and the model learns on this information so as to be summed up to other information later on. It has the test dataset (or subset) so as to test our model's forecast on this subset and it will do this utilizing SciKit-Learn library in Python utilizing the train_test_split technique.

\section{Data Wrangling}

In this section of the report will load in the data, check for cleanliness, and then trim and clean given dataset for analysis. Make sure that the document steps carefully and justify for cleaning decisions.

\section{Data Collection}

The informational index gathered for foreseeing understanding is part into Training set and Test set. For the most part, 7:3 proportions are applied to part the Training set and Test set. The Data Model which was made utilizing guileless baysien calculation are applied on the Training set and dependent on the test outcome precision, Test set expectation is finished.

\section{Preprocessing}

The data which was collected might contain missing values that may lead to inconsistency. To gain better results data need to be preprocessed so as to improve the efficiency of the algorithm. The outliers have to be removed and also variable conversion need to be done. Based on the correlation among attributes it was observed that attributes that are significant individually include tnm, stages, grade, age, which is the strongest among all. Some variables such as applicant income and co- applicant income are not significant alone, which is strange since by intuition it is considered as important.

\section{MODULE DESCRIPTION}

- Data Validation Process

- Exploration Data Analysis of Pre-Processing and Visualization Process

- Outlier Detection Process by Symptom Based Given Attributes

- To Training a Model by Given Dataset Using Sklearn Package

- Accuracy Results of Logistic Regression And Decision Tree Algorithms

- Accuracy Results of Svm, Knn And Naive Bayes

- Selection of Best Algorithm by Additional Parameters

- Gui Based Prediction Results of Heart Stroke / Heart Attack

\section{Description:}

\section{Data Validation Process}

Validation techniques in machine learning are used to get the error rate of the Machine Learning (ML) model, which can be considered as close to the true error rate of the dataset. If the data volume is large enough to be representative of the population, you may not need the validation techniques. However, in real-world scenarios, to work with samples of data that may not be a true representative of the population of given dataset. To finding the missing value, duplicate value and description of data type whether it is float variable or integer. The 
sample of data used to provide an unbiased evaluation of a model fit on the training dataset while tuning model hyper parameters.

\section{Exploration Data Analysis of Pre-Processing and Visualization Process}

Information representation is a significant ability in applied measurements and AI. Insights do surely concentrate on quantitative portrayals and estimations of information. Information perception gives a significant suite of devices for increasing a subjective comprehension. This can be useful when investigating and becoming more acquainted with a dataset and can help with recognizing designs, degenerate data, peculiarities, and fundamentally more. With a little territory data, data recognitions can be used to express and show key associations in plots and charts that are more natural and accomplices than extents of affiliation or importance. Information representation and exploratory information investigation are entire fields themselves and it will suggest a more profound jump into some the books referenced toward the end.

\section{Outlier Detection Process by Symptom Based Given Attributes}

Many machine learning algorithms are sensitive to the range and distribution of attribute values in the input data. Outliers in input data can skew and mislead the training process of machine learning algorithms resulting in longer training times, less accurate models and ultimately poorer results. It couldn't fit the model on the training data and can't say that the model will work accurately for the real data. For this, we must assure that our model got the correct patterns from the data, and it is not getting up too much noise. Cross-validation is a technique in which we train our model using the subset of the data-set and then evaluate using the complementary subset of the data-set.

\section{To Training a Model by Given Dataset Using Sklearn Package}

In python, sklearn is machine learning packages which include a lot of ML algorithms. Here, we are using some of its modules like train_test_split, DecisionTreeClassifier or Logistic Regression and accuracy score.

\section{Accuracy Results of Logistic Regression and Decision Tree Algorithms}

False Positives (FP): An individual who will pay anticipated as defaulter. At the point when genuine class is no and anticipated class is yes. For example in the event that real class says this traveler didn't endure however anticipated class reveals to you that this traveler will endure.

False Negatives (FN): An individual who default anticipated as payer. At the point when genuine class is yes however anticipated class in no. For example in the event that real class esteem shows that this traveler endure and anticipated class reveals to you that traveler will kick the bucket.

True Positives (TP): A person who won't pay foreseen as defaulter. These are the precisely foreseen positive characteristics which infer that the estimation of authentic class is yes and the estimation of foreseen class is similarly yes.

True Negatives (TN): An individual who default anticipated as payer. These are the effectively anticipated negative qualities which implies that the estimation of real class is no and estimation of anticipated class is additionally no. For example on the off chance that genuine class says this traveler didn't endure and anticipated class reveals to you something very similar. 


\section{Accuracy Results of SVM, KNN and Naive Bayes}

K-Nearest Neighbor is a directed AI computation which stores all events contrast with planning data centers in n-dimensional space. Exactly when a darken discrete data is gotten, it analyzes the closest $\mathrm{k}$ number of cases saved and reestablishes the most generally perceived class as the conjecture and for authentic regarded data it reestablishes the mean of $\mathrm{k}$ nearest neighbors. A classifier that orchestrates the enlightening list by setting a perfect hyper plane between data. I picked this classifier as it is unimaginably adaptable in the amount of different kernelling limits that can be applied and this model can yield a high consistency rate. Support Vector Machines are perhaps one of the most acclaimed and examined AI figuring's.

\section{Selection of Best Algorithm by Additional Parameters}

It is important to compare the performance of multiple different machine learning algorithms consistently and it will discover to create a test harness to compare multiple different machine learning algorithms in Python with scikit-learn. It can use this test harness as a template on your own machine learning problems and add more and different algorithms to compare. Each model will have different performance characteristics. Using resampling methods like cross validation, you can get an estimate for how accurate each model may be on unseen data. It needs to be able to use these estimates to choose one or two best models from the suite of models that you have created. When have a new dataset, it is a good idea to visualize the data using different techniques in order to look at the data from different perspectives. The same idea applies to model selection. You should use a number of different ways of looking at the estimated accuracy of your machine learning algorithms in order to choose the one or two to finalize. A way to do this is to use different visualization methods to show the average accuracy, variance and other properties of the distribution of model accuracies.

\section{GUI Based Prediction Results of Heart Stroke / Heart Attack}

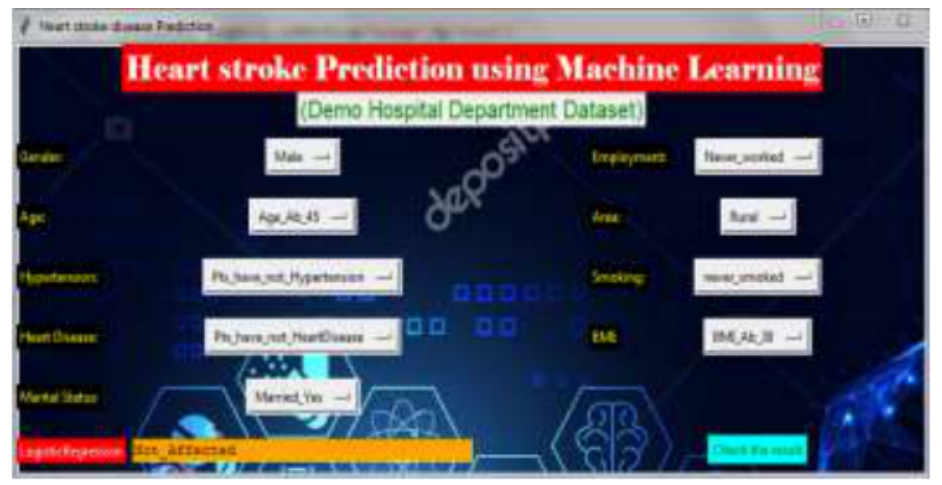

\section{SYSTEM ARCHITECTURE}

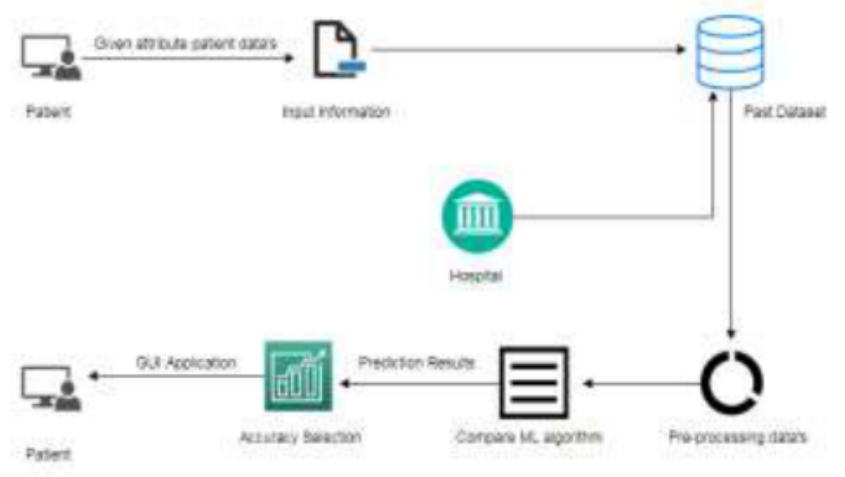




\section{FUTURE ENHANCEMENT}

Emergency focus needs to motorize the seeing of the heart stroke from ability process (consistent) in context on the record detail. To automate this strategy by show the measure bring about web application or work district application. To refresh the work to execute in Artificial Intelligence condition.

\section{CONCLUSION}

The indicative method started from data cleaning and getting ready, missing worth, exploratory assessment in conclusion model structure and appraisal. The best precision on open test set is higher exactness score is will be find. This brings a segment of the going with bits of information about break down the heart stroke disease. Early finding of stroke is commonly huge for the patient to diminish its impact. To give a desire model the guide of automated thinking to improve over human precision and outfit with the degree of early acknowledgment.

\section{REFERENCES}

[1] J. S. Hochman et al., "Early Revascularization and Long-term Survival in Cardiogenic Shock Complicating Acute Myocardial Infarction," JAMA, vol. 295, no. 21, p. 2511, Jun. 2006.

[2] P. a. Heidenreich et al., "Anticipating the eventual fate of cardiovascular illness in the United States: An approach proclamation from the American Heart Association," Circulation, vol. 123, pp. 933-944, 2011.

[3] V. L. Roger, "The study of disease transmission of cardiovascular breakdown," Circ. Res., vol. 113, no. 6, pp. 646-659, 2013.

[4] D. Mozaffarian et al., Heart Disease and Stroke Statistics-2016 Update: A Report From the American Heart Association. 2015.

[5] H. Thiele, E. M. Ohman, S. Desch, I. Eitel, and S. de Waha, "The board of cardiogenic stun," Eur. Heart J., vol. 36, no. 20, pp. 1223-1230, May 2015.

[6] L. Mill operator, "Cardiogenic Shock in Acute Myocardial Infarction the Era of Mechanical Support," J. Am. Coll. Cardiol., vol. 67, no. 16, pp. 1881-1884, 2016.

[7] A. D. Nagpal, R. K. Singal, R. C. Arora, and Y. Lamarche, "Impermanent Mechanical Circulatory Support in Cardiac Critical Care: A State of the Art Review and Algorithm for Device Selection," Can. J. Cardiol., vol. 33, no. 1, pp. 110-118, 2017.

[8] A. Reyentovich, M. H. Barghash, and J. S. Hochman, "The board of obstinate cardiogenic stun.," Nat. Fire up. Cardiol., vol. 13, no. 8, pp. 481-92, Aug. 2016.

[9] P. E. Marik, "Eulogy: aspiratory vein catheter 1970 to 2013.," Ann. Serious Care, vol. 3, no. 1, p. $38,2013$.

[10] H. J. C. Swan, W. Ganz, J. Forrester, H. Marcus, G. Precious stone, and D. Chonette, "Catheterization of the Heart in Man with Use of a FlowDirected Balloon-Tipped Catheter," N. Engl. J. Drug., vol. 283, no. 9, pp. 447-451, Aug. 1970. 
Dr. J.C. Kavitha, K. Jayasurya, Kurakula Premkrishna, R. Praveenkumar

[11] W. Ganz, R. Donoso, H. S. Marcus, J. S. Forrester, and H. J. Swan, "another procedure for estimation of cardiovascular yield by thermodilution in man.," Am. J. Cardiol., vol. 27, no. 4, pp. 392-6, Apr. 1971.

[12] S. N. Ahmed, F. M. Syed, and D. T. Porembka, "Echocardiographic assessment of hemodynamic parameters," Crit. Care Med., vol. 35, no. 8 SUPPL., 2007.

[13] J. A. Chirinos, "Blood vessel firmness: Basic ideas and estimation systems," J. Cardiovasc. Transl. Res., vol. 5, no. 3, pp. 243-255, 2012.

[14] T. J. Iberti, E. P. Fischer, A. B. Leibowitz, E. A. Panacek, J. H. Silverstein, and T. E. Albertson, "A multicenter investigation of doctors' information on the aspiratory corridor catheter. Aspiratory Artery Catheter Study Group," Jama., vol. 264, no. 22, p. 2928-32., 1990.

[15] K. Ikuta, Y. Wang, A. Robinson, T. Ahmad, H. M. Krumholz, and N. R. Desai, "National Trends being used and Outcomes of Pulmonary Artery Catheters Among Medicare Beneficiaries, 1999-2013," JAMA Cardiol., vol. 06520, pp. 1-6, 2017. 